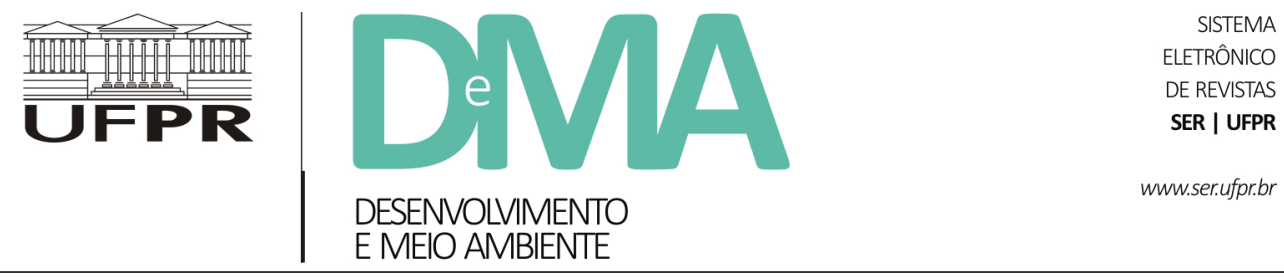

\title{
Evolução dos estudos sobre a produção de bovinos de corte e a emissão de gases de efeito estufa decorrente dessa atividade na região central do Brasil
}

\section{The evolution of studies on beef cattle and emission of greenhouse gases caused by this activity in the central region of Brazil}

\author{
Julhana Dias FACHINETTO ${ }^{1}$, Marlon Vinícius BRISOLA²* \\ ${ }^{1}$ Faculdade Central de Cristalina (FACEC), Cristalina, GO, Brasil. \\ ${ }^{2}$ Programa de Pós-graduação em Agronegócios (PROPAGA), Universidade de Brasília (UnB), Brasília, DF, Brasil. \\ *E-mail de contato: mvbrisola@gmail.com
}

Artigo recebido em 22 de junho de 2016, versão final aceita em 7 de março de 2018.

RESUMO: A preocupação com as mudanças climáticas globais tem se tornado cada vez mais evidente com o aumento de debates e discussões sobre o tema em âmbito internacional. O efetivo bovino brasileiro é o maior rebanho comercial do mundo e emite, aproximadamente, $50 \mathrm{~kg}$ de metano por animal ao ano. Considerando essa significativa participação da pecuária bovina brasileira na emissão de gases de efeito estufa (GEE), este estudo se propôs a analisar os avanços nas pesquisas brasileiras sobre a atividade de produção de bovinos de corte e seu impacto no meio ambiente no decorrer dos últimos 25 anos, na região central do Brasil, onde se concentra o maior efetivo bovino. Foram utilizados como base de dados os documentos publicados pela Embrapa Gado de Corte durante esse período. Como forma de medida do impacto ambiental causado pela atividade, foi utilizada a média de emissão de metano proveniente da fermentação entérica e do manejo dos dejetos dos animais, multiplicada pelo efetivo da região. Uma matriz booleana foi utilizada como instrumento para comparar os períodos e as variáveis. Os resultados encontrados indicaram que os estudos sobre o impacto da atividade pecuária no meio ambiente não contribuíram satisfatoriamente para a redução individual de metano pelos bovinos ao longo do período, mesmo com o aumento considerável da produção de carne bovina.

Palavras-chave: bovinocultura de corte; GEE; tecnologia.

ABSTRACT: Concerns about global climate change has become increasingly apparent with increasing debates and discussions on the subject at an international level.Brazilian cattle effective is the largest commercial herd in the world, emitting about $50 \mathrm{~kg}$ of methane per animal each year. Considering this significant participation 
of Brazilian cattle ranching in the emission of Greenhouse Gases (GHG), the present study aimed to analyze the advances in Brazilian research on beef cattle production activity and its impact on the environment over the past 25, in central Brazil, which concentrates the most effective cattle. Documents published by Embrapa Beef Cattle during this period were used as database. As a way to measure the environmental impact of the activity, we used the average of methane emissions from enteric fermentation and manure management of the animals, multiplied by the effective region. Moreover, a Boolean Matrix was used as a tool to compare periods and variables. The results indicate that studies on the impact of livestock farming on the environment did not respond satisfactorily to the individual reduction of methane by cattle over the period, regardless of the considerable increase in beef production.

Keywords: beef cattle; GHG; technology.

\section{Introdução}

O Brasil é o quinto maior país do mundo em extensão territorial, com cerca de $20 \%$ de sua área ocupada por pastagens, e concentra o maior rebanho bovino comercial do planeta. Apesar de o potencial brasileiro para a produção agropecuária contribuir para o suprimento da demanda mundial por alimentos e biocombustíveis, muitos obstáculos ainda precisam ser vencidos para que tal desafio seja alcançado de maneira sustentável, de modo a gerar não apenas benefícios econômicos para o país, mas também garantir a conservação de sua grande riqueza de recursos naturais e proporcionar melhores condições de vida para as próximas gerações (ABIEC, 2013).

A respeito da produção da pecuária de corte nacional, não se pode desprezar os avanços que ocorreram - e também os que vêm ocorrendo - na busca de uma atividade mais produtiva. Entretanto, sabe-se que essa atividade é uma das mais danosas ao meio ambiente. Diante dessa dualidade, uma pergunta fica no ar: Será que esses avanços na produção são compatíveis com e proporcionais ao acúmulo de conhecimento e à transferência de tec- nologia referentes a uma produção ambientalmente sustentável?

Neste estudo, por meio de uma pesquisa documental, buscou-se identificar a evolução, ao longo dos últimos 25 anos, do pensamento científico acerca da sustentabilidade ambiental, o qual se reflete nas técnicas utilizadas na atividade de produção de bovinos de corte na região que se denominou de Brasil Central. Essa região compreende os estados de Minas Gerais, Mato Grosso, Mato Grosso do Sul e Goiás, que, juntos, detêm $45 \%$ do efetivo bovino nacional. Mediante os achados, confrontou-se os dados em relação ao crescimento do rebanho bovino com os dados da emissão de gases de efeito estufa (GEE) produzidos por estes. Isso foi feito por meio do cálculo do efetivo bovino na região ao longo do período analisado (1990-2014).

Por meio da análise de produções técnico-científicas dos últimos 25 anos, relacionadas ao controle e à sustentabilidade ambiental da produção de bovinos de corte e disponibilizadas por pesquisadores do Centro Nacional de Pesquisa de Gado de Corte (CNPGC), da Empresa Brasileira de Pesquisa Agropecuária (Embrapa), instalado no Estado do Mato Grosso do Sul, procedeu-se a uma classificação temática e uma categorização (por 
objetivos) em uma matriz de escala de intensidade comparada. Com base nesses achados, foram levantadas as conclusões do estudo.

\section{A evolução da pecuária de corte no Brasil}

A pecuária, uma das atividades mais antigas realizadas no Brasil, surgiu no país com o processo de colonização, por volta de 1550 , quando os portugueses trouxeram em suas primeiras viagens animais para tração e consumo local. A partir daí, a criação de gado não parou de crescer no país (Furtado, 1978). Furtado (1978) indica que, no decorrer do tempo, mudanças nos processos de produção ocorreram, principalmente a partir do século XIX, transformando a atividade de subsistência em uma atividade de maior importância econômica, com foco comercial.

Na década de 1960, com o advento da Revolução Verde, ocorreu o aumento da produção agrícola por meio do desenvolvimento de pesquisas em sementes e insumos químicos, do uso intensivo do solo, da especialização da produção e da utilização de máquinas no campo, o que gerou maior produtividade. Na pecuária, tanto de corte quanto de leite, tal movimento proporcionou grandes avanços no desempenho e no crescimento dos rebanhos bovinos no Brasil (Brisola \& Sousa, 2002).

As mudanças no sistema produtivo pecuário acarretadas pela Revolução Verde geraram um passivo ambiental e socioeconômico não previsto. Com o aumento da produtividade, houve o aumento também da degradação ambiental, com desmatamentos e queimadas que resultaram no surgimento de pragas e na alteração e na contaminação do ecossistema (solos, rios, animais e plantas), por meio de fungicidas, agroquímicos e outros componentes químicos utilizados no preparo e no manejo da terra (Grandi \& Brisola, 2009).

A modernização do campo também alterou a estrutura agrária. Pequenos produtores que não conseguiram se adaptar às novas técnicas de produção - e, consequentemente, não atingiram produtividade para competir com grandes empresas agrícolas - se viram obrigados a sair do ramo agropecuário, o que influenciou a superpopulação das cidades.

Brisola \& Sousa (2002) admitem que, após a Revolução Verde, mudanças no comportamento da cadeia produtiva de gado de corte influenciaram o sistema de oferta e demanda no mercado agropecuário. Com o aumento e a intensificação da produção, a pecuária de corte adotou novas tecnologias e tornou-se mais especializada, proporcionando produções em grande escala e excluindo da atividade os produtores que não conseguiam aderir aos novos procedimentos.

Atualmente, um dos grandes desafios para o desenvolvimento brasileiro é manter o crescimento da produção agropecuária e, ao mesmo tempo, reduzir os impactos dessa produção sobre os recursos naturais. Essa preocupação é percebida também nos debates internacionais e nas pressões cada vez maiores da sociedade por um novo modelo de desenvolvimento, que seja capaz de conciliar o crescimento econômico e a conservação do meio ambiente.

Atualmente, o Brasil é o quinto maior país do mundo em área, com 8,5 milhões de $\mathrm{km}^{2}$ de extensão, e cerca de $20 \%$ de sua área (cerca de 174 milhões de hectares) está ocupada por pastagens (ABIEC, 2013). O setor agropecuário é responsável direta e indiretamente por variações nos ciclos hidrológico, climático e na qualidade dos recursos 
naturais do país, uma vez que utiliza principalmente a terra e os recursos naturais como recursos principais em seu processo produtivo.

Em 2013, o Brasil contava com 211.764 milhões de cabeças de gado, o que representou um crescimento de $0,2 \%$ em relação ao ano de 2012. Esse efetivo, não muito diferente dos números estimados para os dias atuais, pode ser encontrado em todo o território nacional; no entanto, a maior concentração encontra-se na região Centro-Oeste do país $(33,6 \%)$. As demais regiões apresentam concentrações menores, mas não menos significativas: 21,1\% (Norte), 18,6\% (Sudeste), 13,7\% (Nordeste) e $13 \%$ (Sul). Ressalta-se que os dez principais estados produtores de bovinos representam $87,6 \%$ do total efetivo nacional, e Mato Grosso (13,4\%), Minas Gerais $(11,4 \%)$, Goiás $(10,9 \%)$ e Mato Grosso do Sul $(9,9 \%)$ são os estados que representam o maior efetivo de bovinos no IBGE (2013). Devido a esses quatros estados representarem $45,6 \%$ de toda a produção nacional, optou-se pela escolha destes como os objetos deste estudo e por considerá-los, nesta pesquisa, como pertencentes ao "Brasil Central".

\section{Os efeitos da pecuária sobre o meio ambiente}

Segundo Euclides Filho (2000, p. 9), o sistema de produção de bovinos de corte é representado pelo "conjunto de tecnologias e práticas de manejo, bem como o tipo de animal, o propósito da criação, a raça ou grupamento genético e a ecorregião onde a atividade é desenvolvida", levando-se em consideração os aspectos sociais, econômicos e culturais da região e sua possibilidade e capacidade de promover investimentos. Levando-se em conta essa premissa, pode-se considerar que a diversificação e a especialização da atividade estão diretamente relacionadas às tecnologias aplicadas, seja na alimentação dos animais, no melhoramento genético, nos sistemas de manejo ou nas práticas de gestão e infraestrutura de produção.

Contudo, apesar da reconhecida importância da pecuária na produção de alimentos e na geração de renda, atualmente muitas discussões têm girado em torno do impacto ambiental referente às suas atividades. Segundo estudos realizados por Machado et al. (2011), a pecuária brasileira vem sendo alvo de críticas devido à significativa quantidade de GEE emitidos no decorrer de todo o sistema produtivo. De acordo com o autor, essas críticas são fundamentadas nos baixos índices zootécnicos verificados em sistemas de exploração animal baseados em pastagens degradadas ou que se encontram abaixo de seu potencial de produção, em razão do aumento da quantidade de GEE por quilo de carne produzido, mas não refletem a realidade unânime da produção nacional.

Entre os vários grupos de GEE, a agropecuária contribui de maneira significativa com a emissão de três deles: metano $\left(\mathrm{CH}_{4}\right)$, dióxido de carbono $\left(\mathrm{CO}_{2}\right)$ e óxido nitroso $\left(\mathrm{N}_{2} \mathrm{O}\right) . \mathrm{O} \mathrm{CH}_{4}$, além de apresentar potencial de aquecimento global 25 vezes maior que o $\mathrm{CO}_{2}$, tem tempo de vida na atmosfera de 9 a 15 anos (Machado et al., 2011). A produção de $\mathrm{CH}_{4}$ resulta da fermentação anaeróbica da matéria orgânica em ambientes alagados, como os campos de arroz cultivados por inundação, da fermentação entérica dos animais, do tratamento anaeróbico de resíduos animais e da queima de biomassa (Machado et al., 2011).

As informações preliminares do Segundo Inventário Brasileiro de Emissões e Remoções 
Antrópicas de Gases de Efeito Estufa (MCT, 2009) indicam a emissão total de GEE no ano de 2005, que foi de 2.203.362 $\mathrm{Gg}$ de $\mathrm{CO}_{2}$ eq. ${ }^{1}$. Desse total, a agropecuária foi responsável por $22 \%$ das emissões, e a mudança no uso da terra foi responsável por 57,5\%. Já a contribuição da agricultura na emissão de $\mathrm{CH}_{4}$ nacional foi de $71 \%$, composta principalmente pela emissão entérica, com $63,3 \%$ (54,1\% pelo gado de corte, $7,4 \%$ pelo gado leiteiro e $1,9 \%$ por outras espécies), seguida pela emissão do manejo dos dejetos animais confinados, com 5,5\%. As emissões de $\mathrm{N}_{2} \mathrm{O}$ pela agropecuária representam $90,6 \%$ das emissões nacionais desse gás e são provenientes principalmente da adubação nitrogenada, das fezes e da urina dos animais. Apesar da baixa emissão por unidade de área (pelo fato de as áreas de pastagens no país serem muito grandes), a emissão de $\mathrm{N}_{2} \mathrm{O}$ pelas pastagens representa cerca de 39,4\%.

$\mathrm{O}$ nível de emissão de $\mathrm{CH}_{4}$ produzido pelos animais está diretamente ligado à quantidade e à qualidade do alimento ingerido. Dietas adequadas e balanceadas reduzem a produção de $\mathrm{CH}_{4}$ no rúmen. McAllister et al. (1996) identificam como as principais variáveis que influenciam na produção de $\mathrm{CH}_{4}$ em ruminantes os fatores nutricionais inseridos na quantidade e no tipo de carboidratos da dieta, os níveis de ingestão de alimento e a presença de ionóforos ou de lipídios nestes. Os autores ressaltam ainda que os fatores metabólicos (taxa de passagem da digesta) e ambientais (ligados à temperatura e ao manejo dos animais), além dos estados fisiológicos (tamanho corporal e principalmente a população de microrganismos ruminais, como protozoários e bactérias), também influenciam na produção de $\mathrm{CH}_{4}$.
A intensificação dos sistemas de produção, bem como a melhoria e o aumento de produtividade por animal, reduz a emissão de $\mathrm{CH} 4$ por unidade de produto (Kg de carne). Mesmo que haja aumento na emissão de $\mathrm{N}_{2} \mathrm{O}$, devido ao aumento no uso de fertilizantes nitrogenados no cultivo de grãos para ração ou na adubação de pastagens, essa emissão é compensada (Berndt, 2010). O aumento do uso de tecnologias nos sistemas de produção na pecuária de corte, como a utilização e a intensificação de aditivos nutricionais, a adubação de pastagens, o melhoramento genético, entre outras, contribuem significativamente na redução de $\mathrm{CH}_{4}$ na atmosfera, por meio do melhor aproveitamento do alimento e da redução da idade de abate (O'Hara et al., 2003).

Pesquisas apontam que a capacidade de armazenamento de carbono em pastagens produtivas é superior à das pastagens encontradas em áreas de vegetação nativa. Quando não se tem o cuidado adequado e o manejo correto das pastagens, estas começam a perder o vigor e entram em estágio de degradação, levando o solo a perder carbono e emitir $\mathrm{CO}_{2}$ para a atmosfera. (Berndt, 2010).

Segundo estudos de emissões de GEE no Brasil, o $\mathrm{CO}_{2}$ e o $\mathrm{CH}_{4}$ são os principais gases provenientes da produção de carne bovina. A maior emissão dessa atividade é o $\mathrm{CO}_{2}$, em decorrência do desmatamento, da degradação e do manejo inadequado das pastagens, que são a principal fonte de alimento do rebanho. Em segundo lugar está o $\mathrm{CH}_{4}$ liberado pelo sistema digestivo (fermentação entérica) dos bovinos e em seus dejetos (Lima et al., 1999; Berndt, 2010; Oliveira 2011). Considerando que o $\mathrm{CH}_{4}$ é um potente gás do efeito estufa, $1 \mathrm{ppmv}$

\footnotetext{
${ }^{1} \mathrm{O} \mathrm{CO}_{2}$ eq é uma medida internacionalmente aceita, que expressa a quantidade de GEE em termos equivalentes da quantidade de $\mathrm{CO}_{2}$. A equivalência leva em conta o potencial de aquecimento global dos gases envolvidos e calcula quanto de $\mathrm{CO}_{2}$ seria emitido se todos os GEE fossem emitidos como esse gás.
} 
(partes por milhões em volume) de $\mathrm{CH}_{4}$ inserido na atmosfera gera $0,2^{\circ} \mathrm{C}$ de aumento na temperatura global, devido à capacidade desse gás de absorver o calor $-\mathrm{o} \mathrm{CH}_{4}$ chega a captar 25 vezes mais calor que o $\mathrm{CO}_{2}$ (IPCC, 2006; Berndt, 2010; Silva, 2013). Reduzir esse gás é uma importante opção para minimizar, em curto prazo, o aquecimento global - fator preponderante da escolha do $\mathrm{CH}_{4}$ como o gás de efeito estufa analisado no presente estudo.

\section{Método}

O conjunto metodológico utilizado nesta pesquisa é uma adequação do método usado por Brisola (2013) em seu estudo sobre o upgrading industrial no Brasil e na Argentina e a análise histórica e comparada da relação entre Estado e associações industriais, com base em casos estabelecidos temporalmente. Vale salientar que a denominação "caso" (que poderia ser entendido como "período") foi inicialmente prescrita por Ragin (1992) e reutilizada por Brisola (2013) para estudos longitudinais. A importância dada para estudos históricos comparados também foi valorizada por Mahoney \& Reuschmeyer, 2006). Conforme argumenta Brisola (2013, p. 74), com base em Mahoney \& Reuschmeyer (2006), os "estudos que utilizam métodos histórico-comparativos representam investigações científico-sociais que utilizam fatos históricos e elementos teóricos para desenvolverem e refinarem conceitos, além de identificarem e avaliarem argumentos causais".

O período histórico em análise neste estudo se refere ao intervalo de tempo entre 1990 e 2014, que corresponde a um período de maior conscientização e importância sobre as questões ambientais na eco- nomia, na política e na sociedade brasileira. Além disso, no Brasil, esse foi um período de realização de importantes eventos sobre as mudanças climáticas, que resultaram em acordos e documentos de extrema relevância para o progresso na resolução dos problemas ambientais enfrentados pelo mundo. Considerando essa realidade, o estudo buscou explicar as mudanças processuais na atividade da pecuária de corte ocorridas no Brasil Central, em relação aos aspectos ambientais comparados nos diferentes períodos, ao longo destes 24 anos. Vale ressaltar, conforme mencionado anteriormente, que o Brasil Central, neste estudo, abrange somente os estados de Mato Grosso do Sul, Mato Grosso, Goiás e Minas Gerais.

Cada um dos cinco casos analisados, circunscritos em um período de cinco anos, compreendeu um volume de informações colhidas de fontes secundárias e que poderiam representar condicionantes de impacto ambiental. Essas fontes foram publicações técnico-científicas desenvolvidas por pesquisadores do CNPGC/Embrapa, as quais foram elencadas com base em sete variáveis independentes: 1) manejo para formação de pastagens (MF); 2) manejo para manutenção de pastagens (MM);3) integração de culturas (IC); 4) manejo reprodutivo/ melhoramento genético (MR); 5) manejo alimentar (MA); 6) estrutura física (EF); 7) sistema de gestão (SG). As publicações consideradas foram os manuais técnicos, as apostilas e os boletins técnicos emitidos pelo CNPGC entre 1990 e 2014.

Segundo Ragin $(1987 ; 1992)$, os casos possibilitam a criação de padrões a partir da comparação e favorecem a interpretação dos dados. A associação e a combinação de um conjunto de padrões preestabelecidos são a base para estudos fundamentados na análise de casos. O autor ressalta ainda que a 
produção de conhecimento, nesses casos, deve ser orientada de maneira generalizada e construída com base em evidências conceituais.

A coleta de dados foi realizada por meio de pesquisa documental. O CNPGC, vinculado à Embrapa e localizado em Campo Grande, capital do Mato Grosso do Sul, foi a única e exclusiva fonte de geração de dados utilizada. Essa escolha se deveu ao fato de o centro ser o polo de pesquisa que envolve a pecuária de corte no Brasil e o responsável por viabilizar soluções tecnológicas sustentáveis para a cadeia produtiva da pecuária de corte, em benefício da sociedade brasileira.

Os materiais analisados foram apostilas desenvolvidas para os cursos oferecidos pelo CNPGC/ Embrapa aos pecuaristas e estudantes de graduação e pós-graduação, além de livros, manuais e documentos oficiais publicados pela própria Embrapa. $\mathrm{O}$ total de documentos investigados somam 32 obras, editadas entre 1990 e 2014.

Uma vez identificados, os dados de interesse foram correlacionados com as variáveis e os períodos (casos) correspondentes. Em seguida, cada caso foi analisado em relação ao grau de intensidade em que apareceram manifestas as argumentações em torno do tema (variável), em seus respectivos documentos.

A técnica utilizada para análise dos dados foi uma adaptação da técnica QCA (Qualitative Comparative Analysis), que permite uma análise comparada de casos, com muitas variáveis e com " $n$ " pequeno ou intermediário. Sua essência analítica está apoiada na regra da concordância e da diferença. A apresentação dos dados se dá por meio de uma matriz booleana, que recebe as informações quantificadas (pelos índices de intensidade), por caso e por variável independente, permitindo uma análise interpretativa e comparativa. Da mesma forma, é alimentada por uma variável dependente (Y) e pelo índice de grau de cobertura proporcional (GCP), que nada mais é que uma média aritmética dos índices apresentados, por caso. O GCP facilita a comparação dos casos. A matriz booleana contribui, portanto, para uma comparação entre casos e entre variáveis e indica se há alguma variável (ou um conjunto delas) que se apresenta como necessária à maior (ou menor) prevalência de $\mathrm{Y}$.

Todas as informações colhidas foram categorizadas e classificadas em uma escala de intensidade para a evidência de trabalhos científicos, em que 1 indicou "ausência de estudos", 2, "baixa presença de estudos" e 3 , "importante presença de estudos". A variável dependente do estudo (Y) foi representada pelo cálculo da emissão de $\mathrm{CH}_{4}$, em gigagramas $(\mathrm{Gg})$, decorrente do efetivo bovino médio em cada período (caso) na região estudada.

A variável dependente, estipulada na presente pesquisa como variável $Y$, foi obtida pela multiplicação do número do rebanho bovino de cada período (caso) das unidades federativas estudadas (Brasil Central) pela média de emissão de $\mathrm{CH}_{4}$ produzida por unidade de animal correspondente ao mesmo período analisado. A medida utilizada pela emissão de $\mathrm{CH}_{4}$ foi convertida de quilograma (Kg) para gigagrama $(\mathrm{Gg})$, para facilitar a leitura dos resultados.

Para efeito de cálculo do efetivo bovino (variável Y), considerou-se o número médio de animais presentes no total das unidades federativas, em cada período estudado. Assim, no Caso 1 (1990-1994), contabilizaram-se 74.002.801 animais; no Caso 2 (1995-1999), 77.198.743; no Caso 3 (2000-2004), 92.676.952; no Caso 4 (2005-2009), 93.027.486; no Caso 5 (2010-2014), 95.224.133 (IBGE, 2013). 
As estimativas de emissões de $\mathrm{CH}_{4}$ ao longo do período foram baseadas nos dados disponíveis no Segundo Inventário Brasileiro de Emissões e Remoções Antrópicas de Gases de Efeito Estufa, publicado em 2010. Calculou-se a emissão média de $\mathrm{CH}_{4}$ (por fermentação entérica e por manejo de dejetos), por animal e por caso (período) e foram encontrados os seguintes valores: Caso 1 (19901994): $51 \mathrm{~kg} \mathrm{CH} /$ an./ano, Caso 2 (1995-1999): $52 \mathrm{~kg} \mathrm{CH}_{4} /$ an./ano, Caso 3 (2000-2004): $51 \mathrm{~kg} \mathrm{CH}_{4} /$ an./ano, Caso 4 (2005-2009): $51 \mathrm{~kg} \mathrm{CH}_{4} / \mathrm{an}$./ano e Caso 5 (2010-2014): 52kg CH $\mathrm{KH}_{4}$ an./ano.

\section{Resultados e discussão}

As análises e as discussões foram divididas em diferentes tópicos, que seguem a lógica utilizada na pesquisa. Inicialmente, os resultados foram apresentados em cinco seções, que correspondem a cada um dos cinco casos. Em cada uma delas, foram descritos os procedimentos recomendados pelos pesquisadores da Embrapa, em suas publicações, aos pecuaristas. Vale lembrar que o objetivo da pesquisa não é descrever qualquer e todo processo utilizado na produção de gado no Brasil, e sim identificar os procedimentos que interferem ou estão relacionados aos impactos ambientais causados pela atividade. Esses procedimentos foram analisados partindo-se do viés das sete variáveis independentes preestabelecidas na pesquisa.

O cálculo da somatória das duas fontes de emissões de $\mathrm{CH}_{4}$ pelos bovinos da região do Brasil Central é demonstrado como $\mathrm{Y}$, em gigagramas (Gg), na Tabela 1 - uma demonstração da matriz booleana. Para cada caso, também são apresentados, na mesma tabela, os indicativos de intensidade referentes às variáveis independentes do estudo.

A visualização da matriz booleana evidencia a predominância do índice de intensidade 2 dos indicadores, o que demonstra a existência, no período, de evidências de avanços no conhecimento e na aplicação de técnicas minimizadoras de GGE na pecuária de corte no Brasil.

A variável MR foi a que apresentou maior predominância do índice reativo à ausência de informação (1) quanto às práticas sustentáveis nos documentos analisados, principalmente nos casos 1 ,

TABELA 1 - Matriz booleana, índices de intensidade das variáveis independentes (por caso), grau de cobertura proporcional (GCP) e variável dependente Y.

\begin{tabular}{|c|c|c|c|c|c|c|c|c|c|}
\hline \multirow{2}{*}{ Caso } & \multicolumn{8}{|c|}{ Variáveis independentes } & \multirow{2}{*}{$\begin{array}{c}\mathbf{Y} \\
(\mathbf{G g}) \\
\end{array}$} \\
\hline & MF & MM & IC & MR & MA & EF & SG & GCP & \\
\hline 1 & 2 & 2 & 3 & 1 & 2 & 1 & 2 & 1,9 & 1.880 \\
\hline 3 & 2 & 3 & 3 & 2 & 3 & 2 & 2 & 2,4 & 2.361 \\
\hline 4 & 2 & 2 & 3 & 1 & 2 & 2 & 2 & 2,0 & 2.363 \\
\hline
\end{tabular}

*Projeção

(MF: Manejo para formação de pastagens; MM: Manejo para manutenção de pastagens; IC: Integração de culturas; MR: Manejo reprodutivo; MA: Manejo alimentar; EF: Estrutura física de produção; SG: Sistema de gestão). 
4 e 5 . Em contrapartida, no Caso 2, essa mesma variável apresentou índice de intensidade 3. Variações como essa, entre os casos, não foram evidenciadas, indicando inconsistência nos avanços em torno do tema. É possível admitir que os pesquisadores deram pouca importância ao manejo animal como meio para a redução de danos ambientais - possivelmente por interpretarem que mudanças nesse campo produzissem efeitos mínimos na emissão de gases ou que os avanços nesse campo já tivessem sido suficientes. É estranha, contudo, a variação na intensidade de estudos sobre o tema ao longo do tempo. Para tanto, não há uma explicação convincente.

Em contrapartida, a variável IC foi a que expressou o maior índice de intensidade 3, o que indicou maior predominância de estudos sobre o tema. As técnicas recomendadas relativas a essa variável alteravam entre a consorciação de gramíneas-leguminosas, a integração lavoura-pecuária e a integração lavoura-pecuária-floresta, com maior evidência nos casos 1, 3 e 4 .

Em relação às demais variáveis - $\mathrm{MF}, \mathrm{EF}$ e SG -, observou-se predominância pequena, mas constante, de estudos ao longo dos casos analisados. Já as variáveis MM e MA consistiram naquelas com maior evidência e constância ao longo dos casos. Para essas variáveis, os objetos de estudo mais evidenciados foram aqueles sobre a taxa de lotação, adubação de pastagens, consorciação gramíneas-leguminosas, avaliação de adaptação de forrageiras às condições de solo e clima, além de estudos sobre combate às pragas.

A média ponderada entre os índices, identificada como GCP, identificou variação entre 1,9 e 2,4 entre os casos, com maior evidência de estudos para o Caso 3 e menor evidência de estudos para os casos 1 e 5 . Isso indica que foi evidenciada maior ênfase nos estudos sobre processos na pecuária de corte no Brasil, com o objetivo de reduzir a emissão de GEE, no período entre 2000 e 2004.

Em uma avaliação por caso, observou-se que, no Caso 1 (1990-1994), a única variável que apresentou índice de intensidade 3 foi a IC, por meio das recomendações para a consorciação gramínea-leguminosa. Constatou-se também que não havia estudos sobre as variáveis MR e EF.

Para o Caso 2 (1995-1999), todas as variáveis tiveram algum tipo de recomendação que minimizasse os impactos da atividade sobre o meio ambiente. A variável MR foi a única que apresentou índice 3, o que indicou uma preocupação dos pesquisadores em responder às demandas ambientais com o melhor desempenho reprodutivo dos animais - especialmente por meio da alimentação e do balanceamento mineral no período das estações de monta. A variável MM, apesar de apresentar o índice 2, concentrou novos estudos nas áreas de controle de erosão, taxa de lotação, sistemas de adubação de pastagens, combate ao fogo e controle de pragas e plantas invasoras. $\mathrm{O}$ mesmo aparece nos estudos relativos à variável $\mathrm{MF}$, com estudos sobre correção da acidez do solo, busca de forrageiras melhor adaptadas e adequação à quantidade de sementes no plantio.

No Caso 3 (2000-2004), observou-se maior evidência de estudos indicativos do índice de intensidade 3, quando comparado aos demais casos, o que demonstra um crescimento da preocupação dos estudiosos com o impacto da produção pecuária sobre o meio ambiente. Os principais estudos encontrados nesse período foram em relação às variáveis MM, IC e MA. Quanto à variável MM foram encontrados estudos no que tange às técnicas de calagem e adubação nitrogenada, à taxa de lotação, ao combate 
a plantas invasoras, ao uso de terraços e curvas de nível e à recuperação e à renovação de pastagens. Em relação à variável IC, foram encontrados estudos para integração lavoura-pecuária e consorciação gramíneas-leguminosas. Já para a variável MA, foram encontrados estudos para suplementação alimentar e confinamento. Observou-se também significativo aumento dos indicadores para a variável SG, embora ainda pouco represente no cômpito geral. De maneira geral, evidenciou-se nos estudos uma preocupação com o "todo" da propriedade e do sistema de produção, o que mostrou maior atenção em relação à integração de processos e à busca de um maior equilíbrio do sistema.

O Caso 4 (2005-2009) apresentou manutenção equilibrada dos estudos sobre a temática, embora tenha manifestado novamente uma redução dos trabalhos sobre a variável MR. Nesse caso, a única variável que obteve índice de intensidade 3 foi novamente a IC, com recomendações sobre as práticas de integração lavoura-pecuária e integração lavoura-pecuária-floresta. Isso conota a necessidade de inserção dessas técnicas no processo produtivo da pecuária de corte, como fator de melhor aproveitamento e uso da terra, maior rendimento econômico e ampliação da sustentabilidade ambiental do sistema produtivo.

O Caso 5 (2010-2014), apresentou semelhanças com o Caso 4, com apenas uma variável com índice 3: o MM. Em relação a essa variável, encontraram-se intensivos estudos sobre recuperação e renovação de pastagens, adubação de pastagens e taxa de lotação. Práticas como a preservação de áreas permanentes, a preservação de reservas legais e a preservação da biodiversidade também estiveram presentes de forma mais consistente nesse período. A variável EF, que em geral apresentou índice 2 ao longo dos casos, apontou estudos em áreas fundamentais para o equilíbrio sustentável do sistema de produção, anteriormente não expressos, como estudos em relação a alternativas para tratamento de resíduos.

Por meio da avaliação da variável $\mathrm{Y}$, na matriz booleana (Tabela 1), é possível verificar que a bovinocultura de corte contribuiu com o crescimento de $31 \%$ nas emissões de $\mathrm{CH}_{4}$ ao longo dos últimos 25 anos no Brasil Central. Apesar do efetivo bovino também ter crescido nessa região ao longo do período analisado, a emissão de $\mathrm{CH}_{4}$ superou o crescimento do rebanho, que foi de apenas $28,7 \%$ (Figura 1).

Por meio dessa análise comparativa, vê-se a ausência de redução na emissão de $\mathrm{CH}_{4}$ pela bovinocultura de corte, mesmo com a amplificação dos estudos sobre o impacto dessa atividade no meio ambiente. Contudo, nota-se também que a proporcionalidade entre o crescimento do efetivo bovino e a emissão de $\mathrm{CH}_{4}$ é amplificada no último intervalo (entre os casos 4 e 5), mostrando uma coincidência entre a queda do GCP a partir do Caso 4. É sabido que a resposta aos estudos publicados e à aplicação destes (sobretudo na pecuária de corte) não é imediata, mas os intervalos médios de cinco anos permitem fazer inferências.

Esses dados, contudo, não revelam que o aumento da produção de carne bovina ao longo do período, devido às tecnologias empreendidas no setor, superou em muito a média de crescimento do efetivo bovino. Segundo dados calculados a partir da FNP (2015), o crescimento percentual da produção nacional de carne bovina ao longo dos anos que compreendem os casos 3-4 e 4-5 foi de, respectivamente, $31,0 \%$ e $9,2 \%$. Esses percentuais foram sensivelmente superiores aos relativos ao 
crescimento do efetivo bovino $(0,5 \%$ e $3,0 \%)$ e à emissão de $\mathrm{CH}_{4}(0,1 \%$ e 5,5\%). Assim, é possível afirmar que a resposta tecnológica sobre a produção é convincente, embora não seja sobre o efetivo bovino.

Apesar de os estudos sobre o impacto da atividade pecuária no meio ambiente terem avançado e de os grandes achados sobre novas técnicas, processos e métodos para minimizar o passivo ambiental causado pela pecuária terem sido notórios, os resultados demonstram que esses avanços ainda não são suficientes para reter, significativamente ao crescimento do rebanho, a emissão de $\mathrm{CH}_{4}$.
A pesquisa identificou, ainda, que a evolução dos estudos e das técnicas para mitigar os efeitos dos GEE na produção de carne bovina no Brasil foi, em grande parte, direcionada para as pastagens, e não propriamente para o animal. Essa evolução teve, assim, maior impacto na redução de $\mathrm{CO}_{2}$ pela recuperação e pela renovação das pastagens, por meio da retenção desse gás no solo, se comparada à minimização ou redução da emissão de $\mathrm{CH}_{4}$ produzida pelo animal no processo de fermentação entérica e em seus dejetos.

Como a metodologia utilizada para identificar o impacto da atividade pecuária no meio ambien-

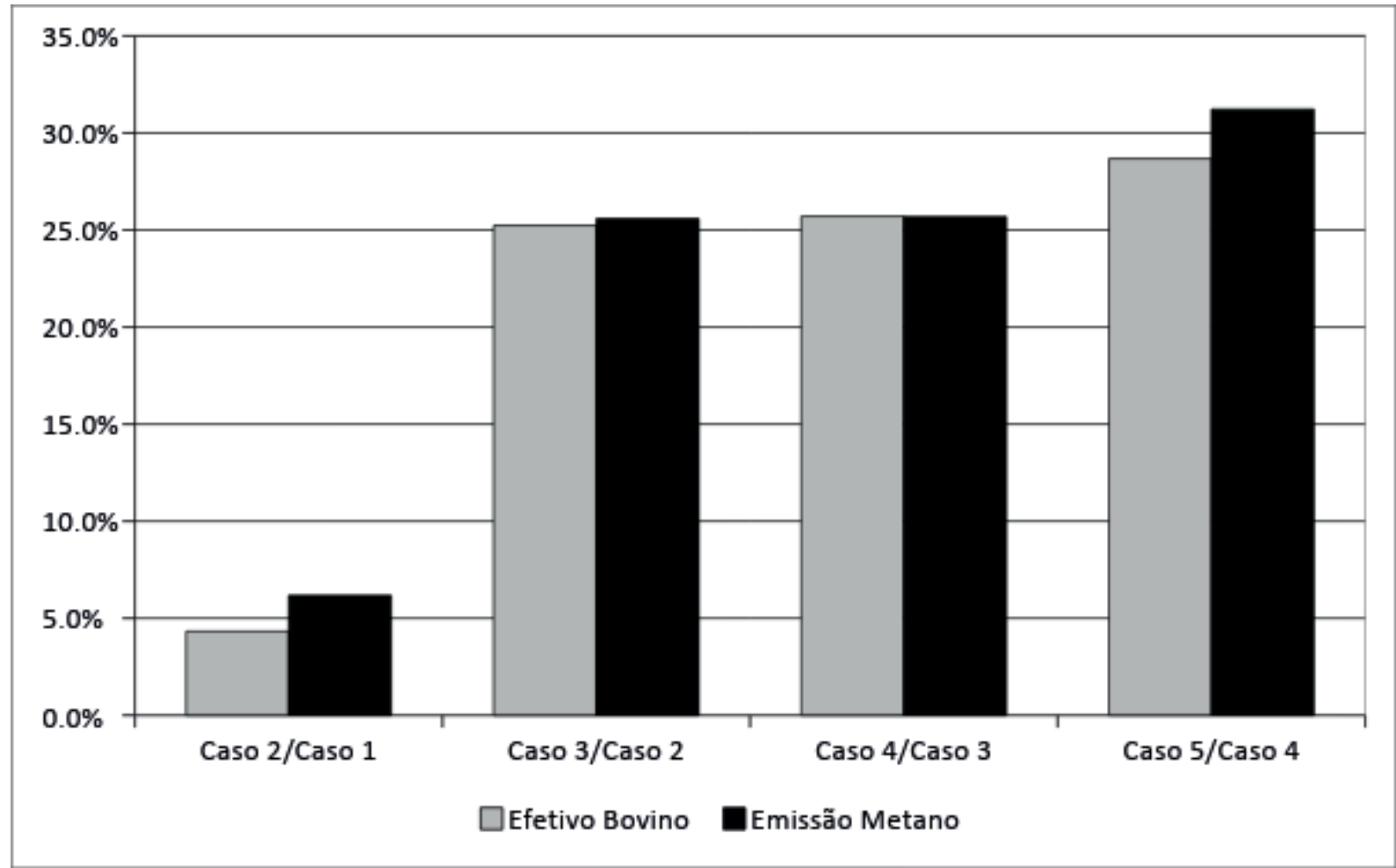

FIGURA 1 - Diferença percentual do efetivo bovino e da emissão de metano, entre os casos analisados, ao longo do período de 25 anos, no Brasil Central.

FONTE: Adaptado (MCT, 2009; FNP, 2015). 
te foi direcionada para estudos sobre a emissão do animal na produção de carne, observaram-se poucos progressos em relação à emissão de $\mathrm{CH}_{4}$. Isso evidencia a necessidade de mais estudos que contribuam para mitigação dos GEE, principalmente do $\mathrm{CH}_{4}$, que é considerado o gás de maior representatividade proveniente da emissão bovina.

As técnicas e os estudos encontrados para mitigação do $\mathrm{CH}_{4}$ pelos bovinos foram relacionados ao melhoramento genético e ao balanceamento alimentar, com o intuito de desenvolver o porte do animal, pois quanto mais eficiente e produtivo for o bovino, menos $\mathrm{CH}_{4}$ será produzido por ele. $\mathrm{O}$ melhoramento genético pode tornar o animal mais resistente e adaptado à região de destino, com uma melhor conversão alimentar. Consequentemente, um animal exposto às condições adequadas alcançará seu ponto de abate mais rápido. Da mesma forma, o balanceamento alimentar possibilita ao animal entrar na fase de acabamento precocemente, pois, quanto mais rápido o boi for abatido, menos $\mathrm{CH}_{4}$ ele emitirá. Há alguns anos, demorava-se de 4 a 5 anos para abater um animal, o que fazia com que a quantidade de emissão de $\mathrm{CH}_{4}$ fosse significativamente maior que a atual, visto que hoje se leva de 2 a 2,5 anos para o abatimento. Essa, talvez, seja a tônica de novos estudos sobre a mitigação dos GEE provenientes da bovinocultura de corte.

Uma ressalva importante a se fazer é o fato de que a evolução dos estudos e procedimentos que levam em conta o equilíbrio do trinômio planta-solo-animal não ocorreu devido a uma preocupação/ consciência ambiental ou a uma responsabilidade socioambiental. Essa evolução ocorreu com o objetivo de aumentar a produtividade do sistema de produção, reduzindo, assim, o custo-benefício da atividade. Contudo, é notório o fato de que, a partir dos anos 2000, a atividade se viu obrigada a se enquadrar nos padrões de qualidade e exigência de uma produção mais sustentável, para reduzir o impacto no meio em que atua.

Nesses 25 anos analisados, é possível identificar uma divisão de águas entre a década de 1990 e os anos 2000. Nos materiais analisados na primeira década, era visível o foco no aumento da produtividade, na expansão do mercado interno para o exterior e na busca por novos conhecimentos para produzir, de modo mais econômico, maiores quantidades de carne, de maneira a gerar uma mais lucratividade ao pecuarista. Apesar de algumas recomendações já terem sido levantadas, ainda era pouco mencionado o impacto da atividade pecuária no meio ambiente em que estava inserida.

A partir do século XXI, a preocupação ambiental se tornou algo evidente em todos os materiais analisados, e o fator sustentabilidade passou a ser crucial para o desenvolvimento e o crescimento da pecuária brasileira. No entanto, os resultados demonstraram que, apesar de os estudos a respeito do impacto da atividade pecuária sobre o meio ambiente terem avançado nas últimas décadas no Brasil, ainda não se mostraram suficientes para reter a emissão de $\mathrm{CH}_{4}$, diante do crescimento do rebanho. Entretanto, a resposta tecnológica existe, e sobre a produção de carne bovina vê-se uma redução percentual significativa da emissão de $\mathrm{CH}_{4}$.

Esta pesquisa indicou que os estudos sobre o impacto da atividade pecuária de corte sobre o meio ambiente revelaram poucos progressos em relação à minimização da emissão de $\mathrm{CH}_{4}$ - gás considerado de maior representatividade proveniente da emissão bovina, proporcionalmente.

Avanços nos estudos sobre o manejo reprodutivo e o melhoramento genético do rebanho 
- considerando o impacto destes sobre a emissão de GEE - seriam desejáveis para a mitigação da emissão de $\mathrm{CH}_{4}$ pelos bovinos. Contudo, esses estudos não foram substancialmente identificados no período analisado, quando comparado com às demais variáveis estudadas.

\section{Conclusão}

O estudo em questão teve como objetivos levantar a produção técnico-científica realizada em um intervalo de 25 anos (de 1990 a 2014) pelo CNPPC/Embrapa e comparar os conteúdos neles abordados com o avanço da emissão de GEE, em razão do efetivo bovino presente nas unidades federativas da região central do Brasil, consideradas suas maiores produtoras. Os períodos de tempo utilizados para comparação foram identificados como "casos" e se fizeram em intervalos de 5 anos. Tal procedimento permitiu a adequada interpretação da relação entre o avanço da pesquisa e a emissão de GEE.

Os principais achados dão conta de que houve um crescente em termos de estudos correspondentes ao tema, ao mesmo tempo que cresceu a própria emissão de GEE ao longo do período analisado. Contudo, houve uma descontinuidade no ritmo de crescimento da emissão de GEE no período correspondente ao Caso 4 (2005-2009).

A interpretação para tal fato tem suporte em algumas hipóteses: é possível que o efeito da transferência de tecnologia, mais amplamente discutida a partir do Caso 3 (2000-2004), ocorra somente no quinquênio seguinte. Contudo, outra interpretação aceita e complementar indica que determinadas áreas de estudos - e sua correspondente aplicação
- impliquem em meios de contenção da emissão de GEE. Estas áreas seriam manejo para manutenção de pastagens, integração de culturas e manejo alimentar. As três áreas apresentaram maior número de estudos no período correspondente ao Caso 3 (2000-2004), ocasião em que ocorreu uma interrupção no crescimento da emissão de GEE, fato que se confirma com a redução da manifestação de produtos técnico-científicos no período seguinte, bem como com a elevação da emissão de GEE.

Considerando os achados anteriores, é possível afirmar que o método utilizado permite afirmar que a produção técnico-científica produzida pelo CNPGC/Embrapa auxiliou no controle da emissão de GEE pelos bovinos criados na região central do Brasil. Tal indicativo mostra a importância da pesquisa aplicada, bem como sua eficácia, quando desenvolvida em determinados temas.

Somente a continuidade de estudos como este poderá dar a certeza de sua fidedignidade analítica no longo prazo. Fica aqui, portanto, um convite a novos pesquisadores para que repliquem o estudo em novos tempos e espaços, dando a certeza de que é possível garantir a manutenção da produção pecuária em um ambiente de maior sustentabilidade.

\section{Referências}

ABIEC - Associação Brasileira das Indústrias Exportadoras de Carnes. Exportações brasileiras de carne bovina, 2013. Disponível em: <http://www.abiec.com.br $>$. Acesso em 20 jan. 2016.

Berndt, A. Impacto da pecuária de corte brasileira sobre os gases do efeito estufa. In: Simpósio de Produção de Gado de Corte, 7. Viçosa, mai. 2010. Disponível em: <http://www. simcorte.com/index/Palestras/7_simcorte/simcorte8.PDF> Brisola, M. V. Upgrading industrial na Argentina e no 
Brasil: uma análise histórica e comparada da relação entre o estado e as associações empresariais industriais. Brasília, Tese (Doutorado em Ciências Sociais) - Universidade de Brasília, 2013.

Brisola, M. V.; Sousa, J. E. Sociological and cultural profile of the "pecuarista de corte" participating in a strategic alliance in Brazil: a case study. In: BALAS - Anual Conference - Transformations in Latin American. Tampa, 2002.

Euclides Filho, K. Produção de bovinos de corte o trinômio genótipo-ambiente-mercado. Campo Grande: Embrapa Gado de Corte, 2000.

FNP, Consultoria \& Comércio. Anualpec. São Paulo: FNP, 2015.

Furtado, C. Análise do 'modelo'brasileiro. Rio de Janeiro: Civilização Brasileira, 6. ed., 1978.

Grandi, J; Brisola, M. V. Produção rural sustentável: uma análise das práticas adotadas pelas organizações rurais da região noroeste de Minas Gerais. In: Jornadas de Estudios Agrarios y Agroindustriales, 9. Buenos Aires, 2009.

IBGE - Instituto Brasileiro de Geografia e Estatística. Produção da Pecuária Municipal, v. 41, 108p. 2013. Disponível em: <ftp://ftp.ibge.gov.br/Producao_Pecuaria/ Producao_da_Pecuaria_Municipal/2013/ppm2013.pdf>

IPCC - Intergovernmental Panel on Climate Change. Guidelines for National Greenhouse Gas Inventories. Cap. 10: Emissions from livestock and Manure Management. p. 10.1-10.84, 2006.

Lima, M. A.; Boeira, R. C.; Castro, V. L. S. Inventário das emissões de gases de efeito estufa provenientes das atividades agrícolas no Brasil. In: Workshop sobre Mudanças Climáticas Globais e a Agropecuária Brasileira. Campinas, 1999.

Machado, F. S.; Pereira, L. G. R.; Guimarães Junior, R.; Lopes, F. C. F.; Campos, M. M.; Morenz, M. J. F. Emissões de metano na pecuária: conceitos, métodos de avaliação e estratégias de mitigação. Juiz de Fora: Embrapa Gado de Leite, 2011.

Mahoney, J.; Ruschemeyer, D. Comparative-historical analysis: achievements and agendas. In: Mahoney, J.; Ruschemeyer, D. (Eds.). Comparative-historical analysis in the social sciences, New York: Cambridge University Press, 3-38, 2006.

Mc.Allister, T. A.; Okine, E. K.; Cheng, K. J. Dietary, environmental and microbiological aspects of methane production in ruminants. Canadian Journal of Animal Science, 76, 231-243, 1996. Disponível em: <http://pubs.aic.ca/doi/ pdf/10.4141/cjas96-035>

MCT - Ministério da Ciência e Tecnologia. Inventário brasileiro das emissões e remoções antrópicas de gases de efeito estufa: informações gerais e valores preliminares. Brasília, 2009. Disponível em: <http://www.cetesb.sp.gov. br/geesp/docs/brasil/6.pdf>

O’Hara, P.; Freney, J.; Ulyatt, M. Abatement of agricultural non-carbon dioxide greenhouse gas emissions: a study of research requirements. Crown Copyright - Ministry of Agriculture and Forestry, New Zealand, 2003.

Oliveira, P. P. A. Emissão de gases nas atividades pecuárias. In: Simpósio Internacional sobre Gerenciamento de Resíduos Agropecuários e Agroindustriais, 2. Foz do Iguaçu, 2011.

Ragin, C. C. The comparative method: moving beyond qualitative and quantitative strategies. London: University of California Press, 1987.

Ragin, C. C. Introduction: cases of 'whats is a cases?' In: Ragin, C. C.; Becker, H. S. (Eds.). Whats is a case? New York: Cambridge University Press, 1-17, 1992.

Silva, R. O. Eaggle: a linear programming model for optimizing mitigation strategies of greenhouse gases emissions in beef cattle production systems. Campinas, Dissertação (Mestrado em Matemática Aplicada) - Instituto de Matemática, Estatística e Computação Cientifica da Universidade Estadual de Campinas, 2013. 